\title{
In Vitro and Intracellular Activities of Omadacycline against Staphylococcus aureus Isolates
}

\author{
Jacques Dubois*, Maïtée Dubois and Jean-Francois Martel \\ M360, Sherbrooke, Québec, Canada \\ •Corresponding author: Jacques Dubois, M3601223 Quatre-Saisons Sherbrooke, Quebec), Canada; E-Mail: jdubois@m360.ca
}

Received: March 18, 2020; Accepted: May 27, 2020; Published: June 03, 2020

\begin{abstract}
Purpose: Omadacycline is a once-daily Intravenous (IV) and oral aminomethylcycline antibiotic that exhibits in vitro activity against Gram-positive and Gram-negative aerobes, anaerobes and atypical bacteria including many drug-resistant strains. This study investigated the in vitro activity of omadacycline and comparators against 239 resistant Staphylococcus aureus strains.
\end{abstract}

Methods: The in vitro activity of omadacycline and comparators was tested against S. aureus strains including methicillin-resistant (mecA), macrolideresistant (ermA, B or C) and ciprofloxacin-resistant (gyrA and parC) isolates. In addition, the intracellular human monocyte activity of omadacycline and comparators was determined against ATCC S. aureus strains.

Results: Against all resistant strains of $S$. aureus, the in vitro activity of omadacycline (MIC90 $0.25 \mathrm{mg} / \mathrm{L}$ ) was lower than that of other tested antibiotics. Bactericidal activity, defined as a mean growth reduction of $\geq 3 \log 10 \mathrm{CFU} / \mathrm{mL}(\geq 99.9 \%)$, was attained at 24 hours of antibiotic exposure with omadacycline, ceftaroline, levofloxacin, and moxifloxacin at extracellular MICs increasing from 1X MIC to 16X MIC against both Methicillin-Sensitive (MSSA) and Resistant (MRSA) strains of $S$. aureus. Mean intracellular growth reduction of $\geq 2 \log 10 \mathrm{CFU} / \mathrm{mL}(\geq 99 \%)$ was achieved at 24 hours by omadacycline, levofloxacin, and moxifloxacin at MICs increasing from 2X to 16X MIC against intracellular S. aureus strains (MSSA and MRSA).

Conclusion: Based on the results of this study, omadacycline exhibits potent extracellular and intracellular activity against $S$. aureus isolates including methicillin- and ciprofloxacin-resistant strains.

Keywords: Omadacycline, Staphylococcus aureus, MIC, Intracellular activity, Tetracycline

\section{Introduction}

Staphylococcus aureus is a frequent cause of serious bacterial infections worldwide $[1,2]$. Skin and skin structure infections have increased in recent years, and the most frequent bacteria were $S$. aureus or other Gram-positive bacteria [2,3]. Effective management of serious skin infections often is complicated by antibiotic resistance, in particular Methicillin-Resistant Staphylococcus Aureus (MRSA), which accounts for nearly half of all isolates from skin and skin structure infections in the United States [4]. Community-Acquired Pneumonia (CAP) is the most common infectious disease leading to hospitalization and mortality among all age groups, especially the elderly $[5,6]$. While $S$. aureus only is isolated in approximately $2 \%$ of cases of CAP, identification of $S$. aureus as a cause of CAP is associated with poor outcomes and increased mortality [1] and has been reported to be the cause of co-infection in 39\%-45\% of patients hospitalized with influenza [7].

Infections due to $S$. aureus often are slow to respond to antibiotics with frequent recurrences and higher mortality [8]. While often thought of as an extracellular pathogen, with inherent problems of antibiotic resistance, new evidence indicates that $S$. aureus is a facultative intracellular pathogen $[8,9]$, and the combination of antibiotic-resistance and intracellular activity can result in incomplete eradication of $S$. aureus even with recommended treatment. Intracellular antimicrobial activity may be markedly impaired compared to in vitro activity observed in broth or extracellular media. Thus, assessing both the intracellular and extracellular activity of antibiotics against $S$. aureus is essential to fully characterize its potential use to treat infections.

Omadacycline, a novel once-daily Intravenous (IV) and oral aminomethylcycline antibiotic, is a semisynthetic tetracycline derivative that exhibits in vitro activity against a range of Grampositive and Gram-negative aerobes, anaerobes, and atypical bacteria $[10,11]$. In vitro and in vivo studies demonstrated that omadacycline circumvents the efflux and ribosomal protection mechanisms of tetracycline resistance and has activity against pathogens common in community-acquired infections, including MRSA [12-14]. In addition, evidence from healthy subjects showed that alveolar cell concentrations of omadacycline exceeded plasma concentrations [15].

This study investigated the in vitro activity of omadacycline and comparators against 239 resistant $S$. aureus including methicillinresistant (mecA), macrolide-resistant (ermA, B or C) and ciprofloxacinresistant (gyrA and parC) strains. In addition, the extracellular and intracellular human monocyte activity of omadacycline and comparators against a variety of ATCC $S$. aureus strains including drug-resistant isolates was determined. 


\section{Methods}

\section{Drugs}

Standard antimicrobial reference powders were provided by the following sources: omadacycline (Lot \#CA16-0193) from Carbogen Amcis AG, Bubendorf, Switzerland; ceftaroline from IRIX Pharmaceuticals, Durham, North Carolina; telithromycin from Sanofi Aventis, Montréal, Québec, Canada; doxycycline, tigecycline, linezolid, levofloxacin, moxifloxacin, azithromycin and erythromycin from Sigma Chemicals, Mississauga, Ontario, Canada.

\section{Strains}

The strain collection represents a phenotypically and genotypically well-characterized variety of resistant community and hospitalacquired S. aureus strains isolated from 1995 to 2016. Twenty of theses 239 (8\%) strains were collected between 1995-2001 and the remainders have been more recently collected during the 2000s.

All strains were grown on Trypticase soy agar (with 5\% sheep blood) to produce pure cultures. Genomic DNA was isolated as previously described [16] and multiplex polymerase chain reaction was performed with primers specific for mecA, ermA, ermB, ermC, and mefE [17] or for gyrA and parC [18]. Four ATCC S. aureus strains (two methicillin-sensitive S. aureus (MSSA) strains (ATCC 29213, ATCC 25923) and two methicillin-resistant (MRSA) strains (ATCC 33591, ATCC 43300)) were also used to assess the extracellular and intracellular activity.

\section{In Vitro Activity}

The in vitro activity of omadacycline was compared with that of doxycycline, tigecycline, linezolid, ceftaroline, levofloxacin, moxifloxacin, telithromycin, azithromycin, and erythromycin against a total of 239 resistant $S$. aureus by broth microdilution according to Clinical and Laboratory Standards Institute (CLSI) guidelines $[19,20]$. The tested strains included $S$. aureus that were methicillinresistant (mecA [150]), macrolide-resistant (ermA, B or C [50]), and ciprofloxacin-resistant (gyrA and parC [39]).

Freshly cation-adjusted Mueller-Hinton broth (Becton Dickinson, Cockeysville, MD, USA) supplemented by $2 \% \mathrm{NaCl}(\mathrm{MH})$ was used as broth medium against resistant $S$. aureus strains, ATCC $S$. aureus strains and Quality Control (QC) strain. MIC microplates, containing approximately $5 \pm 3 \times 105 \mathrm{CFU} / \mathrm{mL}$ in $\mathrm{MH}$ broth and drug dilutions were incubated at $35 \pm 2^{\circ} \mathrm{C}$ in aerobic conditions and were read after 20-24 hours of incubation. Exclusively to simulate the extracellular and phagolysosomal environments, MIC microplates of MH broth were prepared at $\mathrm{pH} 7.4 \pm 0.1$ (original $\mathrm{pH}$ of medium) and $5.5 \pm 0.1$ (modified $\mathrm{pH}$ of medium adjusted with $2 \mathrm{~N} \mathrm{HCl}$ solution) and were tested only against $S$. aureus ATCC strains (ATCC 29213, ATCC 25923, ATCC 33591 and ATCC 43300)).

The minimum inhibitory concentration (MIC) was defined as the lowest concentration of drug that completely inhibited visible growth after incubation. S. aureus ATCC 29213 was included as a QC strain. For extracellular and intracellular activity, MICs obtained at $\mathrm{pH} 7.4 \pm 0.1$ were considered for choosing the tested concentrations.

\section{Determination of extracellular activity}

Kill curve experiments against 4 ATCC $S$. aureus strains (ATCC 25923, ATCC 29213 and ATCC 33591, ATCC 43300) were performed in duplicate by broth microdilution methodology modified from CLSI procedure [21] using flat cell culture microplates. One hundred and fifty microliters of RPMI 1640 medium (with 10\% fetal calf serum) with antimicrobial concentrations of 1 to 6 times their MIC was inoculated with log-phase culture of each ATCC $S$. aureus to final bacterial density of $5 \pm 1 \times 105 \mathrm{CFU} / \mathrm{mL}$ ) into each well of culture microplates for a final volume of $300 \mu \mathrm{L}$. The bacterial cultures were maintained under stationary conditions for 24 hours at $37 \pm 2$ oC in $5 \%$ $\mathrm{CO} 2$ and $95 \%$ air. Counts of $\mathrm{CFU} / \mathrm{mL}$ were perf $\neg$ ormed on all bacterial cultures at time $0,2,6$, and 24 hours of incubation in triplicate using Brain Heart Infusion (BHI) agar.

\section{Determination of intracellular human monocyte activity}

The intracellular activity of omadacycline was compared against 4 ATCC S. aureus strains (ATCC 25923, ATCC 29213, ATCC 33591 and ATCC 43300). The in vitro method using mononuclear cells [22-24] was performed in duplicate using 48-flat cell well culture microplates using RPMI 1640 medium (with $10 \%$ fetal calf serum) and mononuclear cells (THP-1 (ATCC TIB-202) cell line; $2 \pm 1$ X106 cells/ $\mathrm{mL}$ ). Logarithmic-phase culture in BHI broth pelleted down at 14000 r.p.m. for $4 \mathrm{~min}$ and opsonized by suspending pellets in RPMI 1640 supplemented with non-decomplemented $10 \%$ fresh human serum for $30 \mathrm{~min}$ at $37 \pm 2 \mathrm{oC}$. Opsonized $S$. aureus were adjusted to $5 \pm 1 \mathrm{X} 105 \mathrm{CFU} / \mathrm{mL}$ in RPMI 1640 and phagocytized at a 4:1 ratio of bacteria to THP- 1 monocytes. After a 1 hour exposure at $37 \pm 2 \mathrm{oC}$ in a shaking incubator, the infected cultures were centrifuged (1300 rpm; $8 \mathrm{~min}$ ) to eliminate non-phagocytized bacteria and were re-suspended in RPMI medium. One hundred and fifty microliters of RPMI with diluted antibiotics at 1,2,8 or 16 times the MIC of each ATCC $S$. aureus strain were added at time 0 into each well of infected culture microplates for a final volume of $300 \mu \mathrm{L}$. Cultures were maintained under stationary conditions thereafter for 24 hours at $37 \pm 2 \mathrm{oC}$ in $5 \%$ CO2 and $95 \%$ air. Monocytes in a $20 \mu$ sample taken at each time point from each well were diluted by 10 -fold dilutions and lysed with distilled water. Counts of $\mathrm{CFU} / \mathrm{mL}$ at time $0,2,6$, and 24 hours were performed on all bacterial cell cultures in triplicate using BHI agar.

\section{Cytotoxicity}

The cytotoxicity of omadacycline and comparators was assessed in THP-1 monocytes. After $24 \mathrm{~h}$ exposure to antibiotics, even the highest tested concentration (16XMIC) resulted in $<1 \%$ cells being stained with tryptan blue. This observation suggested that all of the tested antibiotics were non-cytotoxic to THP-1 monocytes.

\section{Results}

\section{In vitro Activity}

Against all resistant strains of $S$. aureus, the activity of omadacycline (MIC90 $0.25 \mathrm{mg} / \mathrm{L}$ ) was more potent than other tested antibiotics (Table 1). An MIC90 of $0.25 \mathrm{mg} / \mathrm{L}$ was obtained against methicillinresistant $S$. aureus (mecA genotype group) with omadacycline that was 
Table 1: Susceptibility of resistant $S$. aureus strains: methicillin-resistant (mecA), macrolide-resistant (ermA, B, C), and ciprofloxacin-resistant (gyrA and parC) strains using $\mathrm{MH}$ broth.

\begin{tabular}{|c|c|c|c|c|}
\hline \multirow{2}{*}{ Organism (no. tested) } & \multirow{2}{*}{ Antibiotic } & \multicolumn{3}{|c|}{$\mathrm{MIC}^{\mathrm{a}}(\mathrm{mg} / \mathrm{L})$} \\
\hline & & Range & $50 \%$ & $90 \%$ \\
\hline \multirow{10}{*}{$\begin{array}{l}\text { S. aureus } \\
\text { All resistant tested strains } \\
\text { (239) }\end{array}$} & Omadacycline & $0.016-1$ & 0.25 & 0.25 \\
\hline & Doxycycline & $0.06-\geq 16$ & 0.5 & 1 \\
\hline & Tigecycline & $0.25-1$ & 0.5 & 0.5 \\
\hline & Linezolid & $0.5-4$ & 1 & 2 \\
\hline & Ceftaroline & $0.06-2$ & 0.5 & 2 \\
\hline & Levofloxacin & $0.5-\geq 16$ & 4 & $\geq 16$ \\
\hline & Moxifloxacin & $0.25-\geq 16$ & 4 & $\geq 16$ \\
\hline & Telithromycin & $0.016-\geq 16$ & 0.12 & 4 \\
\hline & Azithromycin & $0.016-\geq 16$ & 2 & $\geq 16$ \\
\hline & Erythromycin & $0.06-\geq 16$ & 1 & $\geq 16$ \\
\hline \multirow{6}{*}{$\begin{array}{l}\text { S. aureus } \\
\text { methicillin-resistant }\end{array}$} & Omadacycline & $0.016-0.25$ & 0.25 & 0.25 \\
\hline & Doxycycline & $0.06-\geq 16$ & 0.5 & 1 \\
\hline & Tigecycline & $0.25-2$ & 0.5 & 0.5 \\
\hline & Linezolid & $0.5-4$ & 1 & 2 \\
\hline & Ceftaroline & $0.06-2$ & 0.5 & 1 \\
\hline & Levofloxacin & $1-\geq 16$ & 4 & $\geq 16$ \\
\hline \multirow[t]{4}{*}{$(150)$} & Moxifloxacin & $0.25-\geq 16$ & 2 & 4 \\
\hline & Telithromycin & $0.016-\geq 16$ & 0.06 & 0.12 \\
\hline & Azithromycin & $1-\geq 16$ & 2 & $\geq 16$ \\
\hline & Erythromycin & $0.5-\geq 16$ & 1 & $\geq 16$ \\
\hline & Omadacycline & $0.06-0.25$ & 0.25 & 0.25 \\
\hline & Doxycycline & $0.25-1$ & 1 & 1 \\
\hline & Tigecycline & $0.25-1$ & 0.5 & 0.5 \\
\hline & Linezolid & $1-4$ & 2 & 2 \\
\hline \multirow{3}{*}{$\begin{array}{l}\text { Macrolide-resistant } \\
\text { ermA, B \& C genotype }\end{array}$} & Ceftaroline & $0.12-2$ & 1 & 1 \\
\hline & Levofloxacin & $0.5-4$ & 2 & 4 \\
\hline & Moxifloxacin & $0.25-4$ & 1 & 4 \\
\hline \multirow{3}{*}{$(50)$} & Telithromycin & $0.12-\geq 16$ & 2 & 4 \\
\hline & Azithromycin & $4-\geq 16$ & $\geq 16$ & $\geq 16$ \\
\hline & Erythromycin & $8-\geq 16$ & $\geq 16$ & $\geq 16$ \\
\hline \multirow{4}{*}{ S. aure } & Omadacycline & $0.06-0.25$ & 0.25 & 0.25 \\
\hline & Doxycycline & $0.5-1$ & 1 & 1 \\
\hline & Tigecycline & $0.25-0.5$ & 0.5 & 0.5 \\
\hline & Linezolid & $1-4$ & 2 & 4 \\
\hline Ciprofloxacin- Resistant & Ceftaroline & $0.06-1$ & 0.5 & 1 \\
\hline \multirow[t]{2}{*}{ gyrA \& parC genotype } & Levofloxacin & $8-\geq 16$ & $\geq 16$ & $\geq 16$ \\
\hline & Moxifloxacin & $4-\geq 16$ & $\geq 16$ & $\geq 16$ \\
\hline \multirow{3}{*}{ (39) } & Telithromycin & $0.016-4$ & 0.06 & 0.25 \\
\hline & Azithromycin & $0.016-\geq 16$ & 0.12 & $\geq 16$ \\
\hline & Erythromycin & $0.12-\geq 16$ & 1 & $\geq 16$ \\
\hline
\end{tabular}

${ }^{a}$ MICs determined by broth microdilution according to CLSI guidelines in antibiotic concentrations from 0.004 to $16 \mathrm{mg} / \mathrm{L}$. Geometric mean value (mg/L) for MIC.

MIC: Minimal Inhibitory Concentration comparable to tigecycline (MIC90 $0.5 \mathrm{mg} / \mathrm{L}$ ), and more potent than doxycycline (MIC90 1mg/L), ceftaroline (MIC90 1mg/L), linezolid (MIC90 2mg/L), and moxifloxacin (MIC90 4mg/L). An MIC90 of $\geq 16 \mathrm{mg} / \mathrm{L}$ was observed with azithromycin, erythromycin, and levofloxacin against methicillin-resistant $S$. aureus (mecA genotype group). Against macrolide-resistant S. aureus (ermA, B, C genotype group) strains, omadacycline (MIC90 $0.25 \mathrm{mg} / \mathrm{L}$ ) was the most active agent and was more active than telithromycin, azithromycin, and erythromycin (MIC90 $\geq 4 \mathrm{mg} / \mathrm{L}$ ) or levofloxacin and moxifloxacin (MIC90 4mg/L). Against ciprofloxacin-resistant S. aureus (gyrA and $\operatorname{parC}$ genotype group), an MIC90 $>16 \mathrm{mg} / \mathrm{L}$ was observed with levofloxacin, moxifloxacin, azithromycin, and erythromycin. While, the MIC90 for omadacycline $(0.25 \mathrm{mg} / \mathrm{L})$ remained lower than that of linezolid (MIC90 4mg/L), doxycycline (MIC90 1mg/L), and ceftaroline (MIC90 1mg/L), this was comparable to telithromycin (MIC90 0.25mg/L) and tigecycline (MIC90 0.5mg/L).

At pH 7.4, the MICs obtained for omadacycline against ATCC $S$. aureus strains (ATCC 29213, ATCC 25923, ATCC 33591 and ATCC 43300 ) were from 0.25 to $0.5 \mathrm{mg} / \mathrm{L}$ (Table 2). Against the four tested ATCC S. aureus strains, the MICs of omadacycline were comparable to tigecycline (MIC range: 0.12 to $0.5 \mathrm{mg} / \mathrm{L}$ ) and ceftaroline (MIC range: 0.12 to $2 \mathrm{mg} / \mathrm{L}$ ). At a pH of 5.5 (phagolysosomal environments), omadacycline MICs were one to two 2-fold serial dilutions higher against ATCC $S$. aureus strains, which was less active than ceftaroline but comparable to tigecycline, linezolid, levofloxacin, and moxifloxacin.

\section{Extracellular and Intracellular Activity}

Bactericidal activity, defined as mean growth reduction of $\geq 3 \log 10$ $\mathrm{CFU} / \mathrm{mL}$ ( $\geq 99.9 \%$ ), was reached at 24 hours of antibiotic exposure with omadacycline, ceftaroline, levofloxacin, and moxifloxacin at increasing extracellular MIC concentrations from $1 \mathrm{X}$ to $16 \mathrm{X}$ MIC against both MSSA and MRSA ATCC strains (Figures 1-4). At 6 hours, growth reduction ( $\geq 2 \log 10 \mathrm{CFU} / \mathrm{mL}$ or $\geq 99 \%$ ) of $S$. aureus (MSSA and MRSA) was detected at $1 \mathrm{X}$ to $16 \mathrm{X}$ MIC with omadacycline and moxifloxacin, at $1 \mathrm{X}$ to $8 \mathrm{X}$ (data not shown) MIC with ceftaroline, and at $8 \mathrm{X}$ (data not shown) to 16X MIC with linezolid. At 24 hours, growth reduction of S. aureus (MSSA and MRSA) was detected with linezolid at 2X to 16X MIC. Among the tested antibiotics, tigecycline and azithromycin only demonstrated a bacteriostatic activity (growth reduction $<2 \log 10 \mathrm{CFU} / \mathrm{mL}$ or $<99 \%$ ) against tested MSSA and MRSA strains.

Important intracellular activity, mean intracellular growth reduction of $\geq 2 \log 10 \mathrm{CFU} / \mathrm{mL}$ ( $\geq 99 \%$ ), was achieved at 24 hours by omadacycline and levofloxacin, at increasing concentrations from $2 \mathrm{X}$

Table 2: Susceptibility of S. aureus ATCC 25923 and ATCC 33591 strains at pH 7.4 and pH 5.5 in MH broth.

\begin{tabular}{|c|c|c|c|c|c|c|c|c|}
\hline \multirow{2}{*}{ Organism tested } & \multirow{2}{*}{$\mathrm{pH}$} & \multicolumn{7}{|c|}{$\mathrm{MIC}^{\mathrm{a}}(\mathrm{mg} / \mathrm{L})$} \\
\hline & & Omadacycline & Tigecycline & Linezolid & Ceftaroline & Levofloxacin & Moxifloxacin & Azithromycin \\
\hline \multirow[t]{2}{*}{ S. aureus ATCC 25923} & 7.4 & 0.5 & 0.12 & 2 & 0.12 & 0.25 & 0.06 & 0.5 \\
\hline & 5.5 & 2 & 0.5 & 4 & 0.12 & 1 & 0.25 & $>16$ \\
\hline \multirow[t]{2}{*}{ S. aureus ATCC 33591} & 7.4 & 0.25 & 0.25 & 2 & 0.5 & 8 & 2 & $>16$ \\
\hline & 5.5 & 1 & 2 & 2 & 0.25 & $>16$ & 4 & $>16$ \\
\hline
\end{tabular}

${ }^{a}$ MICs determined by broth microdilution according to CLSI guidelines in antibiotic concentrations from 0.004 to 16 mg/L. Geometric mean value (mg/L) for MIC.

Only data for strains, S. aureus ATCC 25923 and ATCC 33591 is shown due to similar data for strains S. aureus ATCC 29213 and ATCC 43300. 

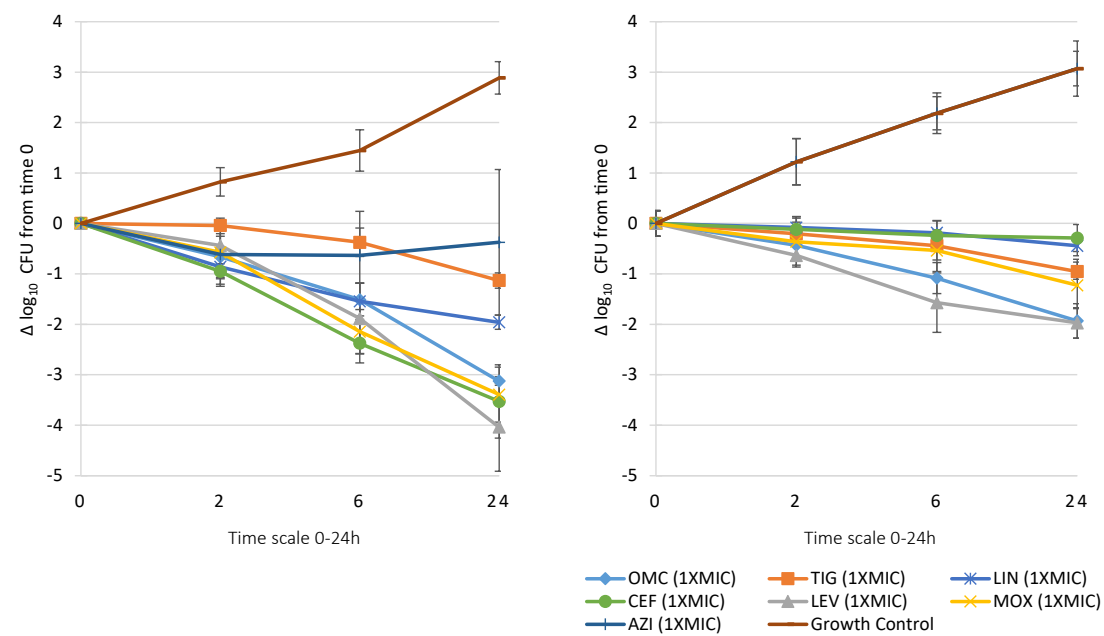

Figure 1: In vitro extracellular (left) and intracellular (right) activity against S. aureus all tested strains: 2 MSSA (ATCC 29213 \& 25923) and 2 MRSA (ATCC 33591 \& 43300 ) with omadacycline (OMC) and comparators (tigecycline (TIG), linezolid (LIN), ceftaroline (CEF), levofloxacin (LEV), moxifloxacin (MOX), azithromycin (AZI)) at 1XMIC from 0-24 hours of incubation. Note that each point corresponds to the mean value of all tested strains determined by triplicate independent counts.
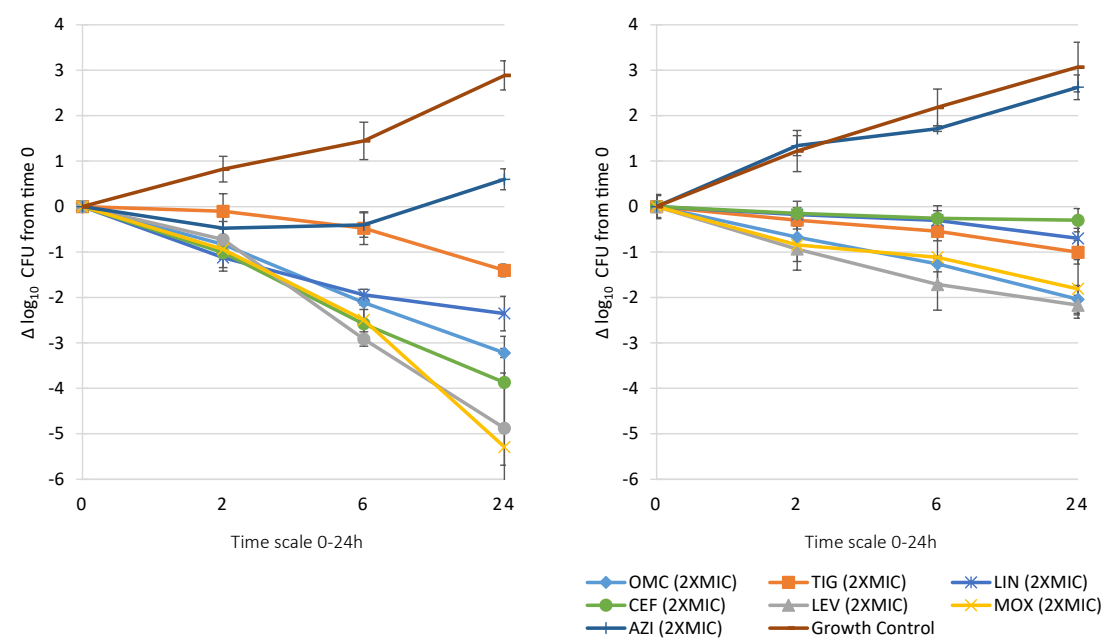

Figure 2: In vitro extracellular (left) and intracellular (right) activity against S. aureus all tested strains: 2 MSSA (ATCC 29213 \& 25923 ) and 2 MRSA (ATCC 33591 \& 43300 ) with omadacycline (OMC) and comparators (tigecycline (TIG), linezolid (LIN), ceftaroline (CEF), levofloxacin (LEV), moxifloxacin (MOX), azithromycin (AZI)) at 2XMIC from 0-24 hours of incubation. Note that each point corresponds to the mean value of all tested strains determined by triplicate independent counts.
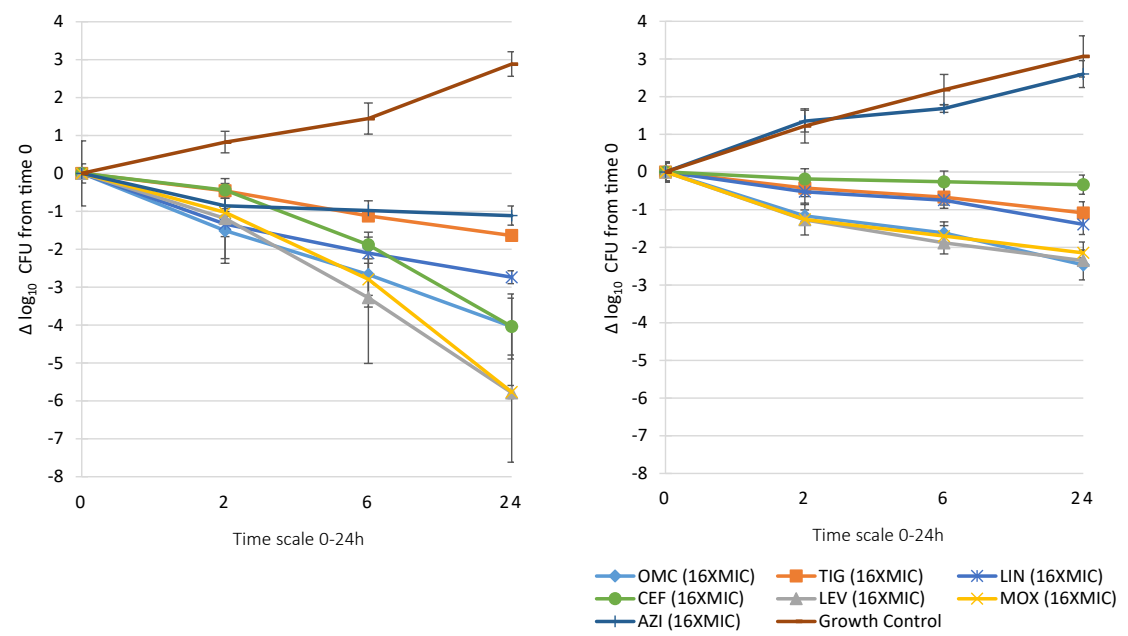

Figure 3: In vitro extracellular (left) and intracellular (right) activity against S. aureus all tested strains: 2 MSSA (ATCC 29213 \& 25923) and 2 MRSA (ATCC 33591 \& 43300) with omadacycline (OMC) and comparators (tigecycline (TIG), linezolid (LIN), ceftaroline (CEF), levofloxacin (LEV), moxifloxacin (MOX), azithromycin (AZI)) at 16XMIC from 0-24 hours of incubation. Note that each point corresponds to the mean value of all tested strains determined by triplicate independent counts. 

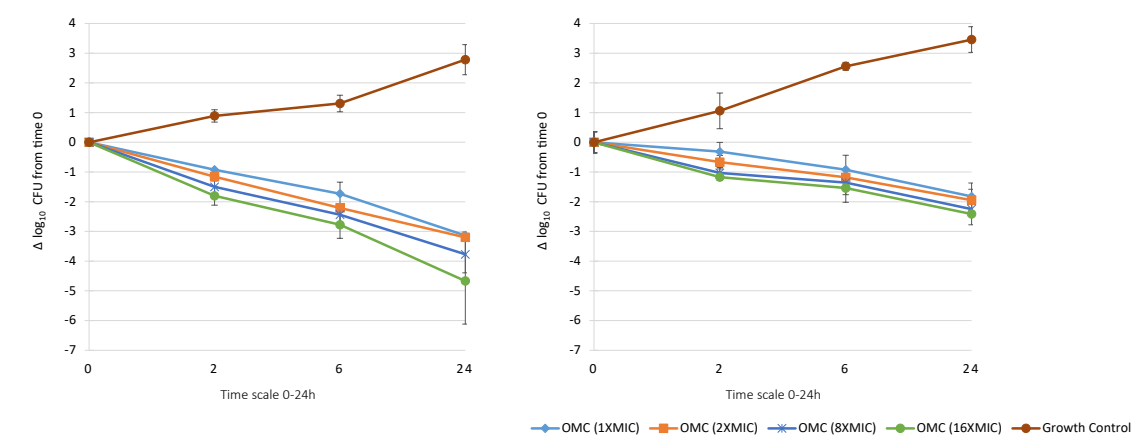

Figure 4: In vitro extracellular activity (left) and intracellular activity (right) against 2 MRSA strains (ATCC 33591 \& 43300) with omadacycline (OMC) at 1X to 16X MIC from 0-24 hours of incubation. Note that each point corresponds to the mean value of 2 MRSA strains determined by triplicate independent counts. Only data of strains MRSA ATCC 33591 and ATCC 43300 is shown due to similar data of strains MSSA ATCC 29213 and ATCC 25923.

to 16X MIC against intracellular MSSA and MRSA (Figures 1-4). At 24 hours, intracellular activity, mean intracellular growth reduction of $\geq 1 \log 10 \mathrm{CFU} / \mathrm{mL}$ ( $\geq 90 \%$ ) but $<2 \log 10 \mathrm{CFU} / \mathrm{mL}$ (<99\%), against intracellular MSSA and MRSA was detected with omadacycline at $1 \mathrm{X}$ MIC, levofloxacin at $1 \mathrm{X}$ MIC, moxifloxacin at $1 \mathrm{X}$ MIC and $2 \mathrm{X}$ MIC, tigecycline at $2 \mathrm{X}$ MIC or greater, and linezolid at $8 \mathrm{X}$ MIC or greater (data not shown). Unlike omadacycline, growth reduction of intracellular MSSA and MRSA was not modified by increasing concentrations of ceftaroline or azithromycin from 1X to 16X MIC.

\section{Discussion}

Results from this study showed that omadacycline exhibits in vitro activity against resistant strains of $S$. aureus. Omadacycline appeared to have more predominant activity than other older tetracyclines, ketolide, macrolides, quinolones, oxazolidinone or third generation cephalosporin's against the most resistant isolates such as $\beta$-lactam-resistant, erythromycin-resistant or ciprofloxacinresistant $S$. aureus. Further, these results revealed that omadacycline has potent extracellular and intracellular activity that was comparable to levofloxacin and moxifloxacin and was higher than tigecycline, linezolid, ceftaroline and azithromycin.

Previously, it was thought that $S$. aureus only infected the extracellular space, and treatment failure was caused by resistance mechanisms to many antibiotics that were inherent in S. aureus [8]. However, in recent years, $S$. aureus were discovered to exist in the intracellular space (phagolysosomes) in macrophages, monocytes, and other human cells as well as the extracellular space, which may provide a more complete explanation for failed antibiotic treatment of infections due to $S$. aureus $[8,9,25]$. Importantly, the results from this study showed that omadacycline demonstrated not only bactericidal extracellular activity (99.9\% of growth reduction) but also produced an intracellular activity (99\% of growth reduction) in human monocytes infected with resistant $S$. aureus. This finding supports the potential clinical activity of omadacycline against a broad variety of $S$. aureus isolates.

The pharmacokinetics of omadacycline has been studied extensively in healthy volunteers after IV and oral administration [15, 26-29].

Recent studies also provided evidence for the presence of omadacycline in alveolar macrophages of animals and humans [15, $25,30]$. The pharmacokinetics of omadacycline and tigecycline were evaluated in plasma, epithelial lining, and alveolar cells of healthy subjects. Subjects received omadacycline $100 \mathrm{mg}$ IV every 12 hours for 2 doses, then $100 \mathrm{mg}$ IV every 24 hours for 3 doses, and concentrations were measured in pulmonary tissues during bronchopulmonary lavage [15]. At the time of bronchoscopy, a mean area under the concentration-time curve (AUC0-24) value of 17.23 $\mathrm{mg} \bullet \mathrm{h} / \mathrm{L}$ and $302.42 \mathrm{mg} \bullet \mathrm{h} / \mathrm{L}$ was observed respectively in Epithelial Lining Fluid (ELF) or in alveolar cells (AC). Combining the observed mean MIC value $(0.5 \mathrm{mg} / \mathrm{L})$ at 24 hours to obtain a mean intracellular growth reduction of $\geq 2 \log 10 \mathrm{CFU} / \mathrm{mL}$, with the observed mean ELF and AC AUC0-24 values, the estimated AUC0-24/MIC ratio in ELF and AC would be $\sim 35$ and $\sim 605$ for tested ATCC strains of $S$. aureus, respectively. Results from these studies indicate potent intracellular omadacycline concentrations and confirm that omadacycline produces AUC0-24 in ELF or AC suggesting achievable level at infection site that far exceed the MICs or the potential intracellular activity for a broad variety of $S$. aureus including resistant isolates included in this study.

Based on the in vitro results of the study reported here, omadacycline exhibits potent extracellular and intracellular activity against MSSA or MRSA. These results combined with extensive in vitro susceptibility studies and pharmacokinetic studies demonstrating consistent systemic exposure after IV and oral dosing are consistent with results from Phase 3 studies. Omadacycline demonstrates consistent efficacy in patients hospitalized with serious infections due to acute Bacterial Skin and Skin Structure Infections (ABSSSI) and CAP caused by $S$. aureus and other causative pathogens [31, 32].

\section{Declarations}

\section{Acknowledgment}

Editorial support in the form of development of the first draft of the manuscript was provided by Richard Perry, PharmD. Editorial support of the revised manuscript was provided by Theresa E. Singleton, PhD, of Innovative Strategic Communications.

\section{Conflicts of Interest}

The author, Jacques Dubois declare conflicts of interest relevant to this study. The authors, Maitée Dubois, and Jean-François Martel declare no conflicts of interest relevant to this study. 


\section{Funding}

This work was supported by Paratek Pharmaceuticals, Boston, MA.

\section{Authors' Contributions}

Jacques Dubois, Maïtée Dubois, and Jean-François Martel contributed equally to this study, and to the review and revision of the manuscript. Author order was determined both alphabetically and in order of increasing seniority.

\section{References}

1. Tong SY, Davis JS, Eichenberger E, Holland TL, Fowler VG (2015) Staphylococcus aureus infections: Epidemiology, pathophysiology, clinical manifestations, and management. Clin Microbiol Rev 28: 603-661. [Crossref]

2. Dryden M, Andrasevic AT, Bassetti M, Bouza E, Chastre J, et al. (2015) Managing skin and soft-tissue infection and nosocomial pneumonia caused by MRSA: a 2014 follow-up survey. Int J Antimicrob Agents 45 Suppl 1: S1-14. [Crossref]

3. Moet GJ, Jones RN, Biedenbach DJ, Stilwell MG, Fritsche TR (2007) Contemporary causes of skin and soft tissue infections in North America, Latin America, and Europe: report from the SENTRY Antimicrobial Surveillance Program (1998-2004). Diagn Microbiol Infect Dis 57: 7-13. [Crossref]

4. Ray GT, Suaya JA, Baxter R (2013) Incidence, microbiology, and patient characteristics of skin and soft-tissue infections in a US. population: a retrospective populationbased study. BMC Infect Dis 13: 252. [Crossref]

5. File TM Jr, Marrie TJ (2010) Burden of community-acquired pneumonia in North American adults. Postgrad Med 122: 130-141.

6. World Health Organization (2014) Antimicrobial Resistance. Global Report on Surveillance. [Crossref]

7. Chertow DS, Memoli MJ (2013) Bacterial coinfection in influenza. JAMA 309: 275282. [Crossref]

8. Sandberg A, Jensen KS, Baudoux P, Bambeke FV, Tulkens PM, et al. (2010) Intra- and extracellular activities of dicloxacillin against Staphylococcus aureus in vivo and in vitro. Antimicrob Agents Chemother 54: 2391-2400. [Crossref]

9. Sendi P, Proctor RA (2009) Staphylococcus aureus as an intracellular pathogen: the role of small colony variants. Trends Microbiol 17: 54-58.

10. Pfaller MA, Huband MD, Rhomberg PR, Flamm RK (2017) Surveillance of omadacycline activity against clinical isolates from a global collection (North America, Europe, Latin America, Asia-Western Pacific), 2010-2011. Antimicrob Agents Chemother 61. pii: e00018-17. [Crossref]

11. Pfaller MA, Rhomberg PR, Huband MD, Flamm RK (2017) Activities of omadacycline and comparator agents against Staphylococcus aureus Isolates from a surveillance program conducted in North America and Europe. Antimicrob Agents Chemother 61. pii: e02411-16. [Crossref]

12. Draper MP, Weir S, Macone A, Donatelli J, Trieber CA, et al. (2014) Mechanism of action of the novel aminomethylcycline antibiotic omadacycline. Antimicrob Agents Chemother 58: 1279-1283. [Crossref]

13. Macone AB, Caruso BK, Leahy RG, Donatelli J, Weir S, et al. (2014) In vitro and in vivo antibacterial activities of omadacycline, a novel aminomethylcycline. Antimicrob Agents Chemother 58: 1127-1135. [Crossref]

14. Tanaka SK, Steenbergen J, Villano S (2016) Discovery, pharmacology, and clinical profile of omadacycline, a novel aminomethylcycline antibiotic. Bioorg Med Chem 24: 6409-6419. [Crossref]

15. Gotfried MH, Horn K, Garrity-Ryan L, Villano S, Tzanis E, et al (2017) Comparison of omadacycline and tigecycline pharmacokinetics in the plasma, epithelial lining fluid, and alveolar cells of healthy adult subjects. Antimicrob Agents Chemother 61 . pii: e01135-17. [Crossref]
16. Smith AM, Klugman KP, Coffey TJ, Spratt BG (1993) Genetic diversity of penicillinbinding protein $2 \mathrm{~B}$ and $2 \mathrm{X}$ genes from Streptococcus pneumoniae in South Africa. Antimicrob Agents Chemother 37: 1938-1944. [Crossref]

17. Sutcliffe J, Grebe T, Tait-Kamradt A, Wondrack L (1996) Detection of erythromycinresistant determinants by PCR. Antimicrob Agents Chemother 40: 2562-2566. [Crossref]

18. González I, Georgiou M, Alcaide F, Balas D, Linares J, et al. (1998) Fluoroquinolone resistance mutations in the parC, parE, and gyrA genes of clinical isolates of viridans group streptococci. Antimicrob Agents Chemother 42: 2792-2798. [Crossref]

19. Clinical and Laboratory Standards Institute (CLSI) (2018) Methods for Dilution Antimicrobial Susceptibility Tests for Bacteria That Grow Aerobically; Approved Standard-Eleventh Edition. CLSI document M07-A11. Wayne, PA.

20. Clinical and Laboratory Standards Institute (CLSI) (2019) Performance Standards for Antimicrobial Susceptibility Testing; Approved Standard - Twenty-ninth Edition. CLSI document M100-S29. Wayne, PA.

21. CLSI (1999) Methods for Determining Bactericidal Activity of Antimicrobial Agents; Approved Guideline. CLSI document M26-A. Wayne, PA.

22. Lemaire S, Van Bambeke F, Mingeot-Leclercq MP, Tulkens PM (2005) Activity of three \{beta\}-lactams (ertapenem, meropenem and ampicillin) against intraphagocytic Listeria monocytogenes and Staphylococcus aureus. J Antimicrob Chemother 55: 897904. [Crossref]

23. Barcia-Macay M, Seral C, Mingeot-Leclercq MP, Tulkens PM, Bambeke FV (2006) Pharmacodynamic Evaluation of intracellular Activities of Antibiotics against Staphylococcus aureus in a model of THP-1 Macrophages. Antimicrob Agents Chemother 50: 841-851. [Crossref]

24. Dubois J, Dubois M (2019) Levonadifloxacin (WCK 771) exerts potent intracellular activity against Staphylococcus aureus in THP-1 monocytes at clinically relevant concentrations. J Med Microbiol 68: 1-7. [Crossref]

25. Lacoma A, Cano V, Moranta D, Regueiro V, Domínguez-Villanueva D, et al. (2017) Investigating intracellular persistence of Staphylococcus aureus within a murine alveolar macrophage cell line. Virulence 8: 1761-1775. [Crossref]

26. Berg JK, Tzanis E, Garrity-Ryan L, Bai S, Chitra S, et al. (2018) Pharmacokinetics and safety of omadacycline in subjects with impaired renal function. Antimicrob Agents Chemother 62. pii: e02057-17. [Crossref]

27. Bundrant LA, Tzanis E, Garrity-Ryan L, Bai S, Chitra S, et al (2018) Safety and pharmacokinetics of the aminomethylcycline antibiotic omadacycline administered to healthy subjects in oral multiple dose regimens. Antimicrob Agents Chemother 62(2). pii: e01487-17. [Crossref]

28. Sun H, Ting L, Machineni S, Praestgaard J, Kuemmell A, et al. (2016) Randomized, open-label study of the pharmacokinetics and safety of oral and intravenous administration of omadacycline to healthy subjects. Antimicrob Agents Chemother 60: 7431-7435. [Crossref]

29. Tzanis E, Manley A, Villano S, Tanaka SK, Bai S, et al (2017) Effect of food on the bioavailability of omadacycline in healthy participants. J Clin Pharmacol 57: 321-327. [Crossref]

30. Lepak AJ, Zhao M, Marchillo K, VanHecker J, Andes DR (2020) In vivo Pharmacodynamic evaluation of omadacycline against Staphylococcus aureus in the neutropenic mouse pneumonia model. Antimicrob Agents Chemother 64. pii: e02058-19. [Crossref]

31. Stets R, Popescu M, Gonong J et al (2017) A phase 3, randomized, double-blind, multi-center study to compare the safety and efficacy of IV to oral omadacycline to moxifloxacin for the treatment of adult patients with CABP (the OPTIC study). Abstr 1883, ID Week, San Diego, California, USA.

32. O'Riordan WA, Green S, Overcash JS (2017) A phase 3 randomized, double-blind, multi-center study to compare the safety and efficacy of oral and IV omadacycline to linezolid for treating adult subjects with ABSSSI (the OASIS study). Abstr. 630. 27 th European Congress of Clinical Microbiology and Infectious Diseases (ECCMID), Vienna, Austria.

\section{Citation:}

Jacques Dubois, Maïtée Dubois and Jean-Francois Martel (2020) In Vitro and Intracellular Activities of Omadacycline against Staphylococcus aureus Isolates. Internal Med Res Open J Volume 5(2): 1-6. 